Hautarzt 2010 $61: 935-937$

DOI 10.1007/s00105-010-2004-x

Online publiziert: 24 . Oktober 2010

(c) Springer-Verlag 2010

\author{
R. Brehler ${ }^{1} \cdot$ B. Wedi ${ }^{2}$ \\ ${ }^{1}$ Ambulanz für Allergologie, Berufsdermatologie und Umweltmedizin, \\ Klinik und Poliklinik für Hautkrankheiten, Universitätsklinikum Münster \\ ${ }^{2}$ Klinik für Dermatologie, Allergologie und Venerologie, \\ Medizinische Hochschule Hannover
}

\title{
In-vitro-Allergiediagnostik in der Dermatologie
}

mit 45,32 EUR vergütet wird. Hierin sind die Kosten für die Testsubstanzen eingeschlossen. Die Vergütung wäre angemessen, wenn der Hauttest tatsächlich so bezahlt werden würde. Regelleistungsvolumen und qualitätsgebundene Zusatzvolumen (QZV) führen in der Regel zu einer deutlich geringeren bzw. gänzlich fehlenden Vergütung. Auch die Bestimmung von allergenspezifischen IgE-Antikörpern ist streng reglementiert; das allergenspezifische IgE wird nach Ziffer 32426 mit 7,10 EUR vergütet, die Höchstgrenze für allergologische Laborleistungen beträgt aber 65 EUR für Erwachsene und 110 EUR für Kinder (bis zum 6. Lebensjahr). Damit können für Erwachsene neben dem Gesamt-IgE rechnerisch nur 8,5 Untersuchungen abgerechnet werden, was für die Abklärung komplexer allergischer Erkrankungen, insbesondere im Bereich der Nahrungsmittelallergie, in der Regel nicht ausreicht.

Allergenspezifische IgE-Antikörper können mit kommerziellen Methoden gegen vermutlich mehr als 8 oo Allergene detektiert werden. Die Qualität der unterschiedlichen Systeme ist verschieden, ebenso die Qualität der einzelnen Allergene. Es muss davon ausgegangen werden, dass für viele auf dem Markt befindliche Allergene keine positiven Seren zur Qualitätskontrolle vorhanden sind. Damit obliegt dem Allergologen die Aufgabe zu entscheiden, gegen welches Allergen die Bestimmung von IgE-Antikörpern im individuellen Fall sinnvoll ist und wie das Ergebnis zu bewerten ist. Unseres Erachtens kann der Allergologe diese Aufgabe nur dann erfüllen, wenn er das Testsystem sehr genau kennt. Die gesundheitspolitischen Bestrebungen, die Abrechnung von Laboruntersuchungen nach dem Kapitel OIII ausschließlich Laborärzten vorzubehalten, erscheint unter diesem Gesichtspunkt kontraproduktiv. Der Allergologe hätte unter diesen Bedingungen keinen Einfluss auf die Bestimmungsmethode, sein direkter Kontakt zum Hersteller des Testverfahrens würde unterbrochen.

Voraussetzung für die In-vitro-Diagnostik in der Hand des Allergologen ist die Durchführung der Tests unter den vorgeschriebenen Laborstandards. Die im Jahr 2009 veröffentlichte Leitlinie der Deutschen Gesellschaft für Allergologie und klinische Immunologie (DGAKI) unter Beteiligung des Ärzteverbandes deutscher Allergologen (ÄDA), der Gesellschaft für Pädiatrische Allergologie und Umweltmedizin (GPA) und der Deutschen Dermatologischen Gesellschaft (DDG) definiert verbindliche Voraussetzungen [1]. In der präanalytischen Phase der Untersuchung sind neben der Indikationsstellung auch die Probenabnahme, Probenaufbereitung, Probenlagerung und Probenhandhabung zu beachten. In der analytischen Phase muss der Test hinsichtlich Reproduzierbarkeit, Sensitivität, Spezifität und technischer Durchführung überprüft werden, was regelmäßige Kontrollen zu Richtigkeit und Präzision der Bestimmungen erfordert. Postanalytisch stehen die Interpretation, Beurteilung und Einordnung des Befundes im Mittelpunkt. Nach der aktuellen Leitlinie ist ein zukünftiges Ziel darüber hinaus die Entwicklung von Konsensusprotokollen zur
(Krankheitsfall =Fall über 4 Quartale) angesetzt werden kann und laut EBM 
Testdurchführung. Für das Labor gelten die allgemeinen Standards der „Guten Laborpraxis“. Die Qualitätssicherung, auch im allergologischen Labor, wird durch die Bundesärztekammer (BÄK) geregelt. Die hierzu erlassenen BÄK-Richtlinien (RiLi$\mathrm{BÄK}$ ) sind für den Anwender absolut verbindlich. Die letzte Richtlinie der BÄK zu Qualitätssicherungsmaßnahmen im Laborbereich sind im Internet unter der Adresse http://www.bundesaerztekammer.de/downloads/Rili-BAeK-Labor.pdf zugänglich [2]. Die Richtlinie gilt auch für Allergologen, die innerhalb ihrer eigenen Praxis Labortests im Rahmen der Patientenversorgung durchführen und interpretieren. Die wichtigsten Aspekte der letzten Änderungen der Richtlinie für den Bereich der In-vitro-Allergiediagnostik werden in der aktuellen Leitlinie zusammengefasst und hier nochmals genannt:

- Umfassendes Dokumentationssystem für die Durchführung der internen und externen Qualitätskontrollen. Dieses lehnt sich im Wesentlichen an die Richtlinien für die Akkreditierung von diagnostischen Laboratorien an. Hierzu zählen u. a.

- Führen eines Qualitätsmanagement-Handbuchs,

- Anlegen von Standardarbeitsanleitungen (SOP) für Tests und Geräte,

- Validierung der eingesetzten Testverfahren,

- Lenkung der Dokumente,

- Klärung von Beschwerden,

- Feststellung von Fehlern und Maßnahmen zu deren Korrektur,

- Durchführung interner Audits.

Wenn auf eine Akkreditierung ver-

zichtet wird, so muss der verantwort-

liche Arzt die Einhaltung der ent-

sprechenden Qualitätsregeln, wie sie in der RiLiBÄK festgehalten sind, sicherstellen, überwachen und kontrollieren.

- Für alle eingesetzten Labortests müssen interne Qualitätssicherungsmaßnahmen durchgeführt werden. Diese erfolgen mit sog. Kontrollproben. Bei der Bewertung von Kontrollproben sind Fehlergrenzen in der Messabweichung zu beachten. Die Berechnung der Fehlergrenzen für einzelne Tests ist in der RiLiBÄK dargestellt. Die Messabweichung des Einzelwerts außerhalb der vorgegebenen Fehlergrenze hat Konsequenzen für die Freigabe der Messergebnisse von Patientenproben.

- Die externe Qualitätskontrolle (Ringversuche) ist ein weiterer essenzieller Aspekt des Qualitätsmanagementsystems. Allerdings wird zwischen sog. RiLiBÄK-pflichtigen und nicht RiLiBÄK-pflichtigen Parametern unterschieden. Derzeit fällt kein Parameter der In-vitro-Allergiediagnostik unter die RiLiBÄK-pflichtigen Untersuchungen. Für die nicht RiLiBÄKpflichtigen Parameter ist gemäß den Gremienbeschlüssen für den Bereich Medizinische Laboratoriums-Diagnostik eine Teilnahme an Ringversuchen mindestens 2-mal jährlich vorgeschrieben.

Für den Verbleib der In-vitro-Allergiediagnostik beim Allergologen ist die Einhaltung der oben genannten Voraussetzungen bei der Erbringung der Laborleistungen essenziell.

Die Auswahl der individuellen Allergene, gegen die IgE-Antikörper bestimmt werden, ist eine Herausforderung. Die meisten der zur Verfügung stehenden Allergene sind Extrakte aus natürlichen Allergenquellen. Extrakte enthalten neben allergologisch relevanten Bestandteilen auch nichtallergenes Material. Unter anderem bestimmen Auswahl des Rohmaterials und Extraktionsmethode die Eignung des Extraktes für den Nachweis einer Allergie gegen die individuelle Allergenquelle.

Von besonderer Bedeutung für die Auswahl von Testallergenen ist das Wissen um Kreuzreaktionen. Rein serologische Kreuzreaktionen basieren häufig auf IgE-Antikörpern, die gegen den CCD-Anteil von Glykoproteinen („cross-reactive carbohydrate determinants") gerichtet sind. Aktuell wird davon ausgegangen, dass diese IgE-Antikörper nur sehr selten für klinische Reaktionen verantwortlich sind.

Zunehmend werden natürliche oder rekombinant hergestellte einzelne Proteine diagnostisch eingesetzt. Ein Vorteil der rekombinanten Allergene ist die gleichbleibende Qualität, die gute Quantifizierbarkeit und Standardisierbarkeit. Allergenextrakte schwanken dagegen in ihrer Zusammensetzung von Hersteller zu Hersteller und Produktcharge zu Produktcharge. In E. coli exprimierte Proteine sind nicht glykosyliert, die Verwendung dieser Proteine verspricht daher eine höhere Spezifität des Tests. Der Nachweis von IgE-Antikörpern gegen Allergenfamilien kann eine bessere Voraussage von klinischen Reaktionen ermöglichen. Auf der anderen Seite setzt die Verwendung von Einzelproteinen in der Allergiediagnostik aber ein erweitertes Wissen über die Bedeutung der einzelnen Proteine voraus. Nur bei Kenntnis der klinischen Relevanz von IgE-Antikörpern gegen ein einzelnes Protein und Wissen über Sequenzund Epitophomologie kann die rationelle Allergenauswahl getroffen werden. Auch bei der Abklärung einer Insektengiftallergie kann die Verwendung nicht glykosylierter Allergenkomponenten hilfreich sein. In welchem Maß die Bestimmung von IgE-Antikörpern gegen Major- und Minorallergene den Erfolg einer SIT gegen Aeroallergene vorhersagen lässt, wird derzeit kontrovers diskutiert.

Auch die Anwendung von zellulären Tests fällt unter die Regelungen zur Qualitätssicherung im diagnostischen Labor, wenn der Test in der Patientenversorgung eingesetzt wird. Zelluläre Testverfahren sind komplexe Methoden und stellen extrem hohe Anforderungen an die Präanalytik und Analytik. Die korrekte Interpretation der Analyse ist nur möglich, wenn entsprechende Kontrollen zur Anwendung kommen. Diese Voraussetzungen limitieren die Anwendung in der täglichen Routine.

Der fehlende Allergienachweis stellt keinen Grund für das Ausweichen auf alternative Diagnostikverfahren dar. Die Bestimmung von IgG- oder IgG4-Antikörpern, insbesondere gegen Nahrungsmittelallergene, wird in Deutschland von verschiedenen privaten Laboratorien propagiert, Allergien sind durch diese $\mathrm{Me}$ thode aber nicht nachzuweisen. Auch im Serum von Gesunden können diese Antikörper häufig detektiert werden [3].

Über die Lektüre der folgenden Arbeiten hinaus sei auch auf die aktuellen Leitlinien verwiesen [Leitlinien der Deutschen Gesellschaft für Allergologie und Klinische Immunologie (Register-Nr. 061), Guidelines International Network (weltweit umfangreichste Leitlinien-Daten- 
bank http://www.g-i-n.net/), AWMF (http://www.leitlinien.net)], in denen zur Auswahl von Testallergenen Stellung genommen und in denen die Durchführung von Hauttests beschrieben wird. Seit dem 01.01.200o sind alle in der gesetzlichen Krankenversicherung tätigen Ärzte und Krankenhäuser gesetzlich verpflichtet, die leitliniengestützten Kriterien bei der Leistungserbringung zu berücksichtigen. Allerdings darf oder muss in begründeten Fällen von Leitlinien abgewichen werden. Auf der anderen Seite ist die Beachtung von Leitlinien aber Voraussetzung für die Kostenerstattung von erbrachten Leistungen durch die Krankenkassen.

Das vorliegende Heft gibt Ihnen einen Überblick über die aktuellen Möglichkeiten der In-vitro-Diagnostik bei Allergien und soll Sie auch für die Brisanz der Diskussion um die Lage der Allergologie in Deutschland sensibilisieren. Die Aufrechterhaltung des kompletten Spektrums der fachärztlichen allergologischen Leistungen ist für die qualifizierte Versorgung der Patienten unabdingbar.

\section{Korrespondenzadressen}

\section{Prof. Dr. R. Brehler}

Ambulanz für Allergologie, Berufsdermatologie und Umweltmedizin, Klinik und Poliklinik für Hautkrankheiten, Universitätsklinikum Münster Von Esmarch-Str. 58, 48149 Münster r-brehler@uni-muenster.de

\section{Prof. Dr. B. Wedi}

Klinik für Dermatologie, Allergologie und Venerologie, Medizinische Hochschule Hannover

Ricklinger Str. 5, 30449 Hannover

wedi.bettina@mh-hannover.de

Interessenkonflikt. Der korrespondierende Autor weist auf folgende Beziehungen hin: B. Wedi: Referententätigkeit für Fa. Bühlmann Laboratories; Drittmittel erhalten von DPC Biermann GmbH. R. Brehler: Vorträge für Phadia und Siemens Healthcare Diagnostics.

\section{Literatur}

1. In-vitro-Allergiediagnostik. AWMF-Leitlinien-Register Nr. 061/017. http://www.leitlinien.net

2. Richtlinie der Bundesärztekammer zur Qualitätssicherung laboratoriumsmedizinischer Untersuchungen. http://www.bundesaertzekammer.de/ downloads/Rili-BAek-Labor.pdf

3. Stapel SO, Asero R, Ballmer-Weber BK et al (2008) Testing for lgG4 against foods is not recommended as a diagnostic tool: EAACI Task Force Report. Allergy 63(7):793-796

\section{Führungskompetenz für Leitende Ärzte im Krankenhaus}

"Es ist eine Erweiterung des Selbstverständnisses zu beobachten" - Der Autor Jens Hollmann im Interview

\section{Lange Jahre galten fast ausschließlich die medizinischen Kompetenzen als aus- schlaggebend für die Besetzung ärztlicher ISBN: 978-3-642- \\ 05264-4 \\ $44,95 €$} Leitungspositionen. Gilt das aus Ihrer Sicht auch heute noch?

- Herausragende medizinische Kompetenzen sind unverändert eine Selbstverständlichkeit. Zunehmend gewinnen aber auch überfachliche Führungsqualifikationen an Bedeutung, um das Abteilungsteam bedarfsgerecht und zukunftsorientiert zu entwickeln und die Leistungsbereitschaft zu stärken. Wer sich heute um eine Position als Leitender Arzt bewirbt, muss damit rechnen, einem Auswahlverfahren unterzogen zu werden, das speziell diese Führungsqualifikationen prüft - und dies haben Ärzte weder im Studium noch in ihrer weiteren fachlichen Ausbildung erlernt.

\section{Haben sich die Herausforderungen an die Führungskompetenz Leitender Ärzte verändert?}

- Ja - und das spüren auch die meisten Chefärzte sehr deutlich. Die Jahre, in denen Assistenzärzte froh waren, eine Stelle zu bekommen und sich jeder Führung fügten, sind vorbei. Heute verzeichnen Kliniken einen Ärztemangel, und die Arbeitsatmosphäre in einer Abteilung spielt eine wichtige Rolle für die Reputation der Klinik in der Ärzterekrutierung.

\section{Die Art der ärztlichen Führung wirkt sich} also auch auf die Mitarbeiterbindung aus? - Natürlich - und dies berührt maßgeblich die ökonomischen Interessen der Klinik. Ärztlichen Nachwuchs gezielt zu entwickeln und zu binden, ist für die Klinikleitung effektiver und effizienter als die Neugewinnung von Personal. Immer mehr Kliniken verlangen daher ein adäquates Situationsmanagement von ihren ärztlichen Führungskräften und investieren gezielt in die überfachliche Führungsausbildung der Leitenden Ärzte.

\section{Können Sie uns Beispiele nennen?}

- Die drei wichtigsten Kompetenzen überfachlicher Natur sind aus meiner Sicht die Fähigkeit, ein Team zielgenau zu entwi-
Führungskompetenz für Leitende Ärzte Motivationsstrategien, Teamführung und Konfliktmanagement im Krankenhaus

Hollmann, Jens

Springer-Verlag GmbH 1st Edition., 2010, XIII, 199 S. 30 Abb., Geb.

ckeln und zu führen, die Motivation und Leistungsbereitschaft der Mitarbeiter passgenau zu fördern sowie Konflikte souverän analysieren und konstruktiv bewältigen zu können.

\section{Wirken sich Führungskompetenzen karrierefördernd aus?}

- Auf jeden Fall; bei meinen Klienten kann ich überwiegend drei Interessenlagen erkennen: Die Klinikleitung will, dass ihre Ärzte kompetent führen und investiert in die Führungsausbildung ihrer Chefärzte; die Leitenden Ärzte bereiten sich mit der Führungsausbildung auf ihren nächsten Karriereschritt vor und die Chefärzte selbst festigen mit diesen Qualifikationen ihre Position und bauen sie aus.

Sie beraten und coachen Leitende Ärzte, welche thematischen Schwerpunkte sind entscheidend?

- Immer mehr ärztliche Führungspersönlichkeiten legen Wert auf ihre Kompetenzen in den Bereichen Teamführung, Motivationsstrategien und Konfliktmanagement. Es ist eine Erweiterung des Selbstverständnisses zu beobachten. Dort, wo die Klinikleitung noch nicht die Weitsicht hat, ihren Leitenden Ärzten ein solches Führungsprogramm zu ermöglichen, werden diese zunehmend selbst aktiv.

\section{Ihr abschließendes Statement?}

- Früher dachten viele Chefärzte, es ginge auch ohne qualifizierte und kompetente Führung. Natürlich geht es auch ohne, aber der nachhaltige Erfolg der Abteilung ist eben nicht sichergestellt oder wird sich sogar gar nicht erst einstellen.

- Weitere Infos www.medplus-kompetenz.de info@medplus-kompetenz.de

Das Interview führte Hinrich Küster, Springer Medizin Heidelberg 